\title{
3DLC: A Comprehensive Model for Personal Health Records Supporting New Types of Medical Applications
}

\author{
Axel Helmer*, Myriam Lipprandt, Thomas Frenken, Marco Eichelberg, and \\ Andreas Hein \\ OFFIS Institute for Information Technology, Oldenburg, Germany
}

Submitted June 2010. Accepted for publication May 2011.

\begin{abstract}
Motivated by the demographic change, many new medical applications are installed in the user's home environment. These applications make use of ambient sensors, enabling new forms of medical care. Personal Health Records (PHRs) are an instrument for the storage, presentation and communication of health related data provided by these applications. But there are still open issues regarding the cooperation between PHRs and the new applications. On the basis of two medical application scenarios, we developed a new model which defines the appropriate level of abstraction of data generated by medical applications to be stored inside the PHR. The model also determines which part of these data is relevant for the clinical decision making process, and how these data should be communicated to physicians. This paper describes the 3DLC model, which uses three dimensions (clinical decision, frequency and context dependence) to determine the type of the data. We further introduce a prototype PHR system that is able to fulfil the requirements of our scenarios.
\end{abstract}

Keywords: personal health records (PHR), assistive technology, medical information systems, home care, data model

\section{INTRODUCTION}

\subsection{Background}

Most industrial countries are steering towards an "ageing society", which can be characterised by three factors [1]. First, the number of the elderly is rapidly increasing. In Germany, the country had about three million residents aged 65 and older in 1910, and more than 13 million in 2000. Second, the percentage of the elderly population is also increasing. From 1910 to 2000, the percentage of citizens aged 65 and older rose from $5 \%$ to $16 \%$ in Germany. Compared to the number of employable residents aged 18 to 64 , the fraction of the elderly rose from $9 \%$ to $25 \%$, indicating that the younger age groups have growing responsibilities in caring for the elderly. The third factor is the increase of people of very old age. The percentage of German residents aged 80 and 
above has increased from $0.5 \%$ to more than $3 \%$ in the period between 1910 and 2000, an increase by more than six times. Models predict further significant changes of societal structure due to increasing life expectancy and the relatively low birth rates. While there are four residents aged 18 to 64 for every senior resident in Germany today, there will only be two in 2030. Furthermore, the number of high-maintenance patients will increase significantly between 2000 and 2040 . Some studies suggest an increase by as much as $70 \%$ [2]. Combined with the decrease of the number of children per family and the increased requirement of mobility by the labour market, shortages in care for the elderly will become increasingly challenging.

\subsection{Motivation}

In addressing the issue of care for the elderly, the research field of "Ambient Assisted Living" (AAL) studies possible applications of information technology integrated into peoples' living environments as means for providing or enhancing support, comfort, security, and care in order to enable elderly residents - as well as younger residents with special needs - to live independently within their familiar home environment for a longer time than possible without the use of assistive technology. This article focuses on a model that helps to characterize the data of these medical applications in the home environment, which are one important part of the overall AAL research field. A number of different issues for ambient-driven medical applications in the home environment need to be addressed, including management of chronic diseases, compensation for functional losses, control of medication intake, detection of adverse events, primary and secondary prevention and long term monitoring of trends.

Such applications use a variety of different sensors with different characteristics in reference to their data volume and sampling rate, connection types (e.g., cable, wireless, push or pull procedure), measurement parameter, measuring unit, data format, sampling rate, configuration mechanisms etc. Further, these applications need to communicate with different types of data sources with different levels of quality and reliability.

The applications also require that information such as prescriptions, training plans, individual limits for vital parameters, etc. are defined and managed by healthcare professionals while being read and used by assistive devices at home. This entails a bidirectional exchange of health related information between a patient's home and a doctor's or hospital's IT infrastructure. Unfortunately, most existing IT systems for health professionals are currently not prepared for this task. They might have capabilities enabling an exchange of information between health professionals (but often even this is missing); however, in the past, the patient's home was simply not seen as a relevant venue for health care that would require integration into the overall health IT. Additionally, longitudinal cross-enterprise Electronic Health Records (EHRs) are not yet available in many countries.

A possible solution to meet the challenge of data integration is the "Personal Health Record" (PHR), a system which allows the use of an IT system under the patient's own control to record and manage health related information. Various definitions for PHRs have been proposed [3]. According to the Markle Foundation, "the Personal Health Record (PHR) is an Internet-based set of tools that allows people to access and 
coordinate their lifelong health information and make appropriate parts of it available to those who need it" [4].

As an enabling technology, PHR is a key factor in empowering patients [5] and will help them to play an "increasingly central" and active role in their own healthcare, as envisioned by the national consensus conference [3]. PHRs have the potential to improve the way the health systems interact with the patient and will help physicians to gain a more complete and balanced view of their patients [3]. Surveys suggest that patients want to use PHRs and believe in their value [6]. PHRs are the only class of electronic health records that could offer certain patient-centered services like drug interaction checks that take into account over-the-counter drugs available without prescription. They enable patients to write medical diaries or to document their training performance at home with the help of new types of vital parameter sensors, which may also be relevant to clinical diagnostics. Furthermore, they have the potential to successfully address the interoperability issues existing in every health system.

The implementation of PHRs for the wide range of ambitious new health related applications creates different challenges: How could the PHR provide sufficient data for existing and upcoming medical applications? On which level of abstraction should the data be stored in the PHR? What are the right forms of presentation for the potential users of the PHR? What data should be communicated and how could interoperability with other IT systems be assured?

The main objective of this paper is to introduce a model for determining the type of data acquired in medical and AAL applications and stored in PHRs. The model categorises data along three dimensions and is thus called three-dimensional layer context (3DLC) model. In addressing the challenges mentioned above, a short description of two typical applications in AAL is presented. These applications serve as examples for new types of medical applications and are used to develop and exemplify the data model. A PHR prototype is also presented which implements the 3DLC model and fulfils the requirements of the described applications regarding data storage and exchange.

\section{METHODS}

We introduce two applications which represent opposite extremes in the spectrum of new medical applications: "tele-rehabilitation training at home" is a purely medical service with clinical characteristics, whereas "activity detection and medical assessment" represents the upcoming new services that rely on sensor data of which the collection is highly dependent on the user's normal living environment. The latter application implies a long-term monitoring through the use of unobtrusive sensors installed in the home environment. For detecting medical issues like the beginning of dementia, trends of a changing behaviour (gait velocity reduction) can be processed. Both applications have been implemented by the authors (as part of a larger team) as technical prototypes. During the requirements analysis phase of the application development, we determined the sensor data relevant to the data processing of each application and the data that must be communicated in each application scenario. We then discussed the scenarios, application functionality and the relevance of the data with 
physicians, psychologists, gerontologists, geriatricians, sociologists and computer scientists involved in the project. Based on the information collected in this process, we developed a model that represents entities of application-generated information on different levels of abstraction.

\subsection{Tele-rehabilitation Training Application}

After an acute cardiac disease or pulmonary transplantation, a stationary rehabilitation program is implemented to help the patient to regain an individually defined degree of fitness and to reduce the relapse rate. A reduction of risk factors such as smoking and overweight, and regular aerobic ergometer bicycle training can help to avoid new cardiac events. One year after the stationary rehabilitation, the cardiologic risk factors deteriorate in most of the coronary patients [7]. This issue may be addressed by implementing the medically supervised rehabilitation phase III after the stationary rehabilitation [8]. Such implementation may be facilitated by a tele-rehabilitation application.

The tele-rehabilitation of patients with chronic diseases such as chronic obstructive pulmonary disease (COPD) or cardiovascular disease takes place in the home environment. Different exercise activities can be performed to increase the physical fitness level. For that purpose, each patient is equipped with an ergometer bicycle, a set of vital parameter sensors for electrocardiogram (ECG), blood pressure (BP), and Saturation of Peripheral Oxygen $\left(\mathrm{SpO}_{2}\right)$ measurements, as well as a processing unit. The mentoring clinic generates an individualised training plan and transmits it to the tele-rehabilitation system for the patient to perform. The plan contains all medical information about the future training sessions, including alarm thresholds for every medical sensor, training method (interval training, heart rate training or load training), training length and number of sessions per week. For all training methods, the ergometer load is specified for each of the three training phases: warm up, load, and cool down.

The tele-rehabilitation system acts on one hand as a gateway to the clinic and on the other hand as an autonomous reaction system that controls the training session. The training control analyzes the training plan and measures data to avoid any cardiac overload. After the training session, a training report is generated that contains all health related data. Thus, constant monitoring of a patient's health is possible. After transmission to the clinic, the report is validated and the training plan is updated as needed.

\subsection{Activity Detection and Medical Assessments Application}

Acute incidences and severe states of chronic diseases are often preceded by less obvious changes in a person's health state such as a reduced self-selected gait velocity. Such changes very often lead to a reduced self-care ability [9] and thus to changes in daily life habits. Early recognition and correct diagnosis of reasons for such changes may help prevent many incidences or delay diseases [10] and need of care. However, in today's health care systems, such diagnostic possibilities are most often not exploited as medical professionals are consulted only after an acute incident has taken place or 
after symptoms of a chronic disease has become obvious to laymen. This leads to increased follow-up costs and decreased quality of life for people affected. In order to improve the current situation, assessments for a long term estimation of medically relevant behaviour of the elderly need to be implemented in their domestic environments, thus integrating them into health care systems. This would enable healthcare professionals to be informed early of abnormal changes in daily habits that may point to changes in the health state, and may enable early diagnosis of various diseases or even prevention of acute incidences. Such integration can only be effectively implemented by application of technology [11].

Technology-supported assessments in the home environment start by collecting information provided by available sensors. Such information are then typically annotated and interpreted according to additional contextual information. In case of mobility related or activity related assessment, such contextual information includes an environment map that contains information on the positions of sensors and their association with objects within the patient's natural environment. It provides the possibility to interpret distances between sensors or to deduce activities related to certain objects. Activity definitions assign sequences of sensor activations to related activities. Several sequences may be used to deduce the same activity with varying probabilities. Activity definitions may also include contextual conditions under which such sequences change. However, recognised activities still need to be interpreted from a medical point of view in order to be useful in providing additional background information to medical professionals. Recognised patterns may have different meanings depending on the personal habits and medical background of the patient. Recognised activities (characterized by their execution frequency and quality) need to be mapped to relevant medical domains based on medical guidelines if necessary.

\subsection{The Three-Dimensional Layer Context (3DLC) Model}

The main purpose of a PHR is to store, present and share health related personal information. To meet the needs for both scenarios described above and other homebased assisting applications, it is important to understand the characteristics of the data acquired in each application scenario, and to decide which of these data should actually be stored in a PHR and can be understood as health related data. The 3DLC model presented in Figure 1 is a tool for characterising data sources in this context.

The 3DLC model distinguishes among three domains involving the production and processing of medical information. In clinical assessments, a diagnosis can be generated during specific episodes of care using diagnostic procedures such as electrocardiogram (ECG), stress test or Magnet Resonance Imaging (MRI). The raw data (images, signals) are important from a medical perspective and need be collected and stored as diagnostic evidence. A supervised assessment includes an execution of medical procedures and assessments (e.g., long term ECG, rehabilitation training) that could proceed in a home environment. This setting allows to perform more measurements and trend analyses, leading to assessments that predict upcoming diseases and help to define further health related activities. However, no immediate diagnoses are derived. By an unsupervised assessment, health related data can be collected in an unobtrusive manner with non- 


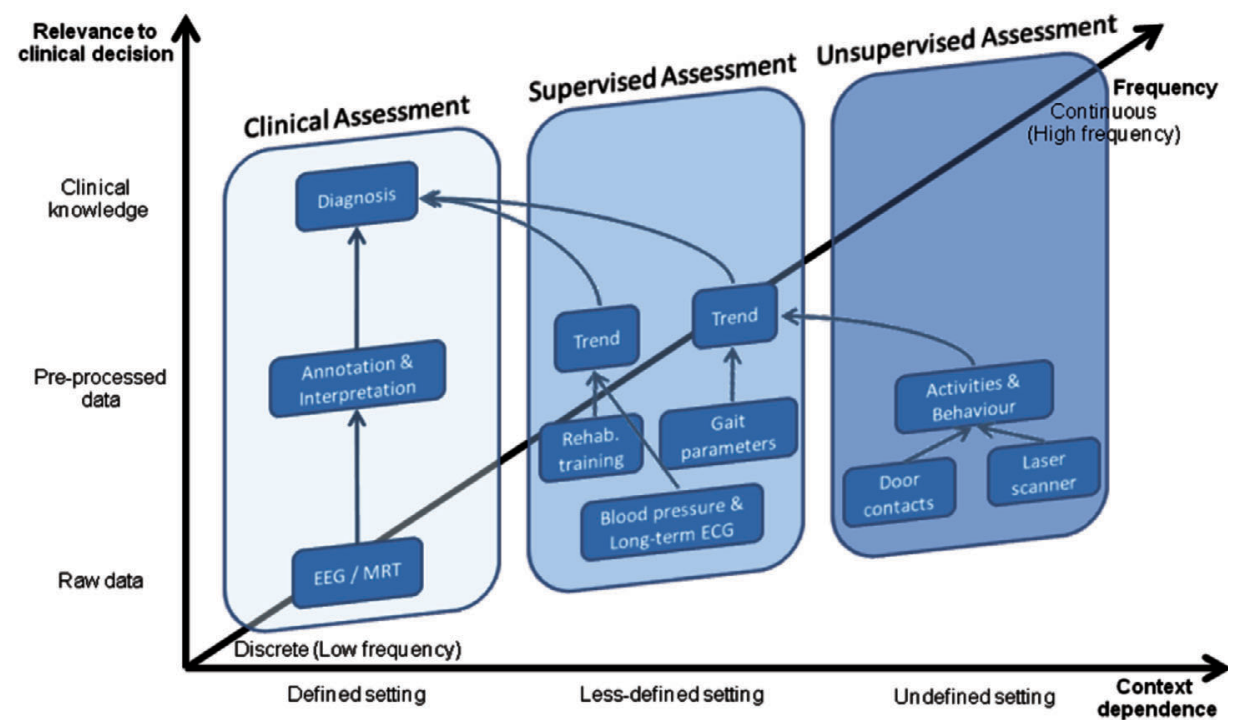

Figure 1. The Three-Dimensional Layer Context (3DLC) Model.

medical techniques and without the patient's aid (e.g., activity detection). The preprocessed data can only be represented as trends for a specific medical question, which could be an early indicator of functional losses.

In case of an unsupervised assessment, the raw data need not be made available to the health professional because the data produced by non-medical sensors like door contacts and laser scanners as such are not meaningful to health professionals; only the trends computed from such data are relevant. Due to the possibility of system failure or the need to check unrealistic medical outcomes (trends), the hardware configuration and raw data should nevertheless be processable afterwards.

The 3DLC model reflects these characteristics in terms of three aspects: context dependence, relevance to clinical decisions (raw data, pre-processed and interpreted medical knowledge), and the frequency in which the data are acquired (discrete vs. continuous), as discussed below.

\subsubsection{Context Dependence}

A generally accepted definition of the term "context" does not exist, although usage of the term is widespread with unspecific meaning. To render the meaning of "context" in 3DLC more clearly, we use the definition of the International Classification of Functioning, Disability and Health (ICF) [12], which is largely about relationships between a person and his or her background of living. Two components or factors can be distinguished: (1) an external factor that reflects environment, such as social structures, and (2) the internal factor that reflects habits of living or psychological states.

The context dependence in our model reflects the unification level of an environment. According to the ICF definition, we adopt the idea of determination 
between a person's capacity (evaluated in a test environment) and the performance in a natural environment. Furthermore, we expand it to a third level of context setting that is located between the two extremes of a fully controlled environment (defined clinical setting) and undefined setting, e. g., the home environment.

The most structured and defined setting for the generation of health related data is the highly standardised clinical environment where high quality data (ECG, MRI) are generated. This provides an evaluation of a person's capacity at a certain time instant and reduces the combination of variables that could influence the outcome. By virtue of clinical tests, decision making is possible. However, the performance of clinical tests usually requires afore-noticed suspicions of medical problems. Furthermore, these tests can only provide a snapshot of a person's state of health.

The less defined context refers to an execution of medical procedures and assessments in a home environment, including activities such as tele-rehabilitation training, blood pressure tests, and long-term ECG. Through this less defined setting, the sensor quality and other influences such as dizziness and time of day are not controlled, thus make the interpretation of sensor data uncertain. The prediction and decision making become more difficult than in a controlled environment.

The undefined setting is a totally unstructured environment and is the routine of many AAL applications. Health related data can be collected in an unobtrusive manner with non-medical techniques and without the patient's aid (e.g., activity detection). For that, the application needs a patient model that reflects the performed and the predicted behaviour.

\subsubsection{Frequency}

The interrelation with the situation in which a patient is performing a medical procedure influences the derivation of medical information out of the sensor data obtained, even if it is an unconscious medical assessment during daily activities. The more undefined the context dependence is, the more measurements are needed to identify reliable trends. This means that data gains more value through the substitution of single point measurements with trend analysis based on more frequent measurements.

The acquisition of vital parameters can be described by the frequency of measurements (high or low sample rate) and the discrete or continuous aspect, i. e. the question of whether monitoring of the subject takes place permanently or only at discrete points in time. The frequency of the data is highly dependent on the context. An undefined context needs a continuous tracking of data for decision making. A less defined context gives alternatives in the granularity of measurements like a point-based blood pressure measurement, or a 24-hour ECG (Holter), which has a high sample rate but is non-continuous. In a clinical environment, the frequency is usually discrete and strictly limited by the time a person could (or would) stay in the clinic.

\subsubsection{Relevance to Clinical Decision Making}

In the "Relevance to Clinical Decision Making" dimension, the abstraction and importance of sensor data can be separated into three layers (see Figure 2). The raw data (Figure 2, bottom) layer is represented through a finite inventory of symbols and 


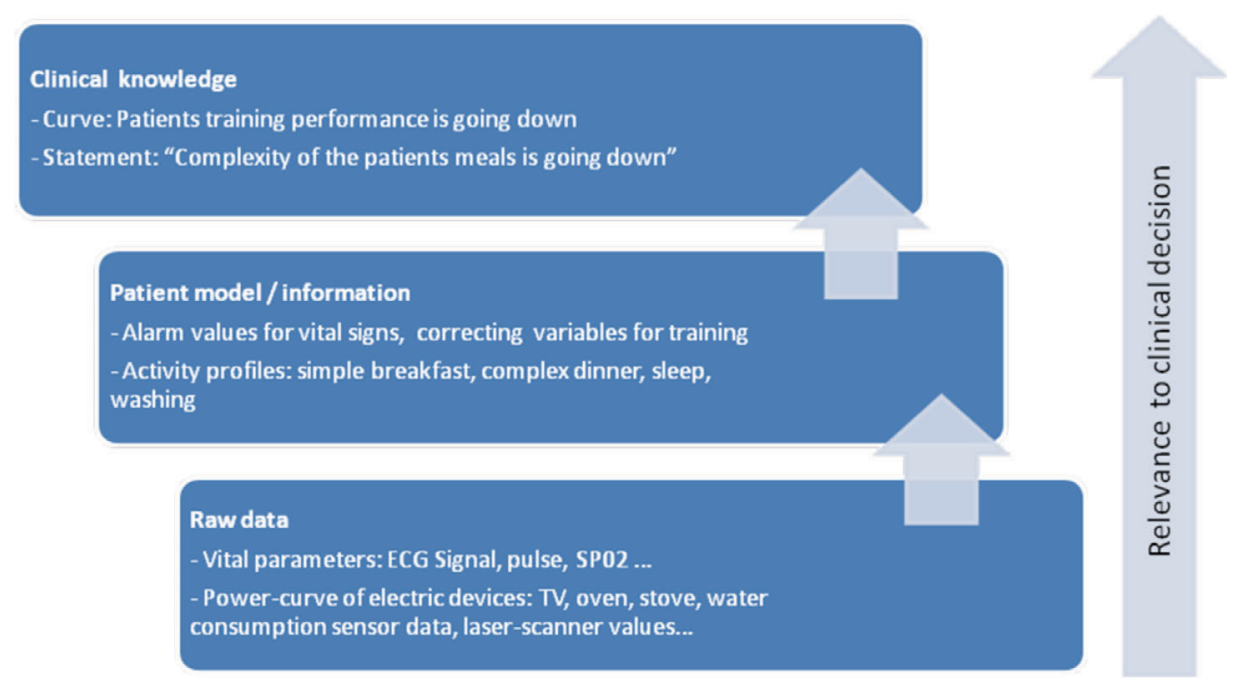

Figure 2. Relevance of information to the clinical decision making process

rule artefacts which convey information. The raw data have to possess structural properties to be interpretable. A pre-processed data element is interpreted using medical knowledge, and taking the current environment into account. Depending on the context, the interpretability of data decreases with the loss of structural and environmental knowledge. In a fully controlled environment, pre-processed data is annotated with medical information. In a less defined or undefined environment, pre-processed data can only be represented as trends for a specific medical question. The analysis of the patient model (Figure 2, center), which could, for example, include the analysis of trends, can lead to concrete clinical knowledge such as: "The patient's training performance is going down" (Figure 2, top), and support a diagnosis. In most cases, the information becomes more important for physicians to be involved with an increasing level of abstraction. The patient model has to be normalised in order to remove artefacts, and ideally also to abstract it from underlying sensor techniques. For example, it does not matter for the clinical decision making process whether a patient has a higher risk for falling is based on information from an accelerometric sensor, or a visual analysis from a camera. Furthermore, the aggregation of sensor data on a higher level of abstraction should lead to more robust and reliable information, rendering it even more valuable to the decision making.

\section{EXEMPLARY APPLICATIONS OF THE MODEL}

This section describes the results of the technical prototype implementation of the two scenarios introduced above (sections 2.1 and 2.2) as well as the adaptation of the data characterisation and the formal model. The integration of both applications and the application of the 3DLC model motivated the adoption of the prototype PHR system. 


\subsection{Tele-rehabilitation Training}

The main challenge in implementing this scenario is the adaptation of the model from laboratory conditions to the tele-rehabilitation system at home. The context characteristics of a clinical setting offer complete control and knowledge about the patient's health status during the training phase. This knowledge makes progress monitoring, emergency detection and adaptation of exercise level possible. Due to the less defined context in a home environment, the core requirement for this application is a system which controls the ergometer load based on vital parameters such as data of ECG, blood pressure (BP), or saturation of peripheral oxygen $\left(\mathrm{SpO}_{2}\right)$, and given alarm thresholds.

In most cases the raw data does not contain sufficient medical information to justify storage in a PHR. Sensor data from home automation devices (such as activity detectors with door contact and power consumption sensors) have little relevance for physicians and patients. Only pre-processed data (such as aggregated curves for activity) containing an adequate level of medical knowledge should be stored in a PHR. An exception is the raw sensor data such as those of ECG and blood pressure. ECG raw (voltage) data can only support the clinical decision process with the help of a dedicated ECG viewer. For that purpose, the raw data must be stored in the PHR because they are a part of the pre-processing layer. Concerning the medical relevance, the raw data from vital sensors in case of the tele-rehabilitation training are of prime importance to derive medical decisions. In contrast, the command and control raw data that increase or decrease the load from the ergometer are not relevant for medical decisions and can be omitted.

With regard to the training report, a patient's fitness trend can be established. Due to the regular training frequency and the a-priori knowledge from the clinic, changes in the health status can be detected. This training report is stored in a medical standard document format called Clinical Document Architecture (CDA) [13, 14]. The main target group for this training report is clinical staff who interpret the information of the performed training session with regard to a changing fitness level or upcoming cardiologic problems.

The properties of CDA meet the requirements of the 3DLC model. The CDA is divided into a three-level architecture. Level 1 represents human readable content only. In levels 2 and 3, this content is extended by machine interpretable codes up to a level of granularity where data are completely machine processable.

The content of a training report includes the ergometer load, the Borg value, the vital parameters of a training session, and the alarms raised. According to 3DLC, only preprocessed data are of medical interest, but the raw sensor data are also stored in the training report. Every training report contains a graphical representation of a training session. This figure exhibits the ergometer load in combination with the vital parameters. Furthermore, aggregated vital parameters, such as average, minimum and maximum heart rate and raised alarms, are represented in the human readable section. In level 3 "entries", the raw sensor data are stored for further processing. The human readable part in the training report reflects the pre-processed level of information from the 3DLC model and the raw data are represented in the fully structured level 3 of CDA. 


\subsection{Activity Detection and Medical Assessments}

Two approaches to mobility and activity monitoring have been implemented, and are currently being evaluated in our research projects. The first approach utilizes a laser range scanner which may be attached to any object or wall in a domestic environment. Installed in the room at approximately leg height, the sensor continuously measures the distance from a person to the sensor within its scan range. The raw range data gathered may be used to precisely and reliably compute various spatio-temporal parameters of human gait such as the self-selected gait velocity [15]. According to the 3DCL model, this approach is classified as clinical assessment. Context dependence is less defined than in clinical environments but not undefined since the assessment is inspired by clinical gait analysis and demands placement of the sensor within a certain height range. The frequency is much higher than in a clinical environment but the data acquisition is not continuous since the assessment can only take place within a limited scan range of a single sensor. Generally, the relevance to clinical decision making is high since the approach computes standardised parameters of human gait. On the other hand, relevance is limited because of the partially uncontrolled domestic environment in which data acquisition takes place, which may cause a reduced data quality compared to acquisition in a well-defined laboratory environment. Additionally, in order to correctly interpret the information collected, additional background information about the patient's health may be required. Nevertheless, the approach delivers clinically relevant information with high frequency directly from domestic environments. In order to increase relevance to clinical decision making, computed parameters have to be subjected to trend analysis or alarm filtering, and thus have to be stored in a PHR along with relevant background information required for further interpretation of the assessment results.

The second approach is classified as home assessment. Sensors from the field of home automation such as light barriers and motion sensors are utilised in order to recognise repetitive motion or activity patterns. In our approach, an environmental map and activity pattern definitions are used to annotate sensors' raw data. According to the 3DLC model, this approach is classified as a totally undefined context, because in this setting, there is often only little knowledge about the environment. However, background knowledge about a patient's habits is essential for interpretation of recognised patterns. This issue is addressed to some degree by the continuous nature of data acquisition, which allows for a long term trend analysis and, based on the recognized patterns, the generation of clinical knowledge relevant to decision making.

\subsection{PHR Architecture and Conceptual Approach}

To face the requirement of a highly available data source for new medical applications, we have chosen a fresh approach to the architectural design of our PHR system. Instead of keeping the information needed by the home-based medical applications on an internet server, we chose to install a set-top box as hardware platform in the user's home as the physical habitat for our system (see Figure 3). The system runs on a set top box and collects data from the home environment (rehabilitation training and activity detection sensors). The raw data are filtered, stored in a database (DB) and processed 


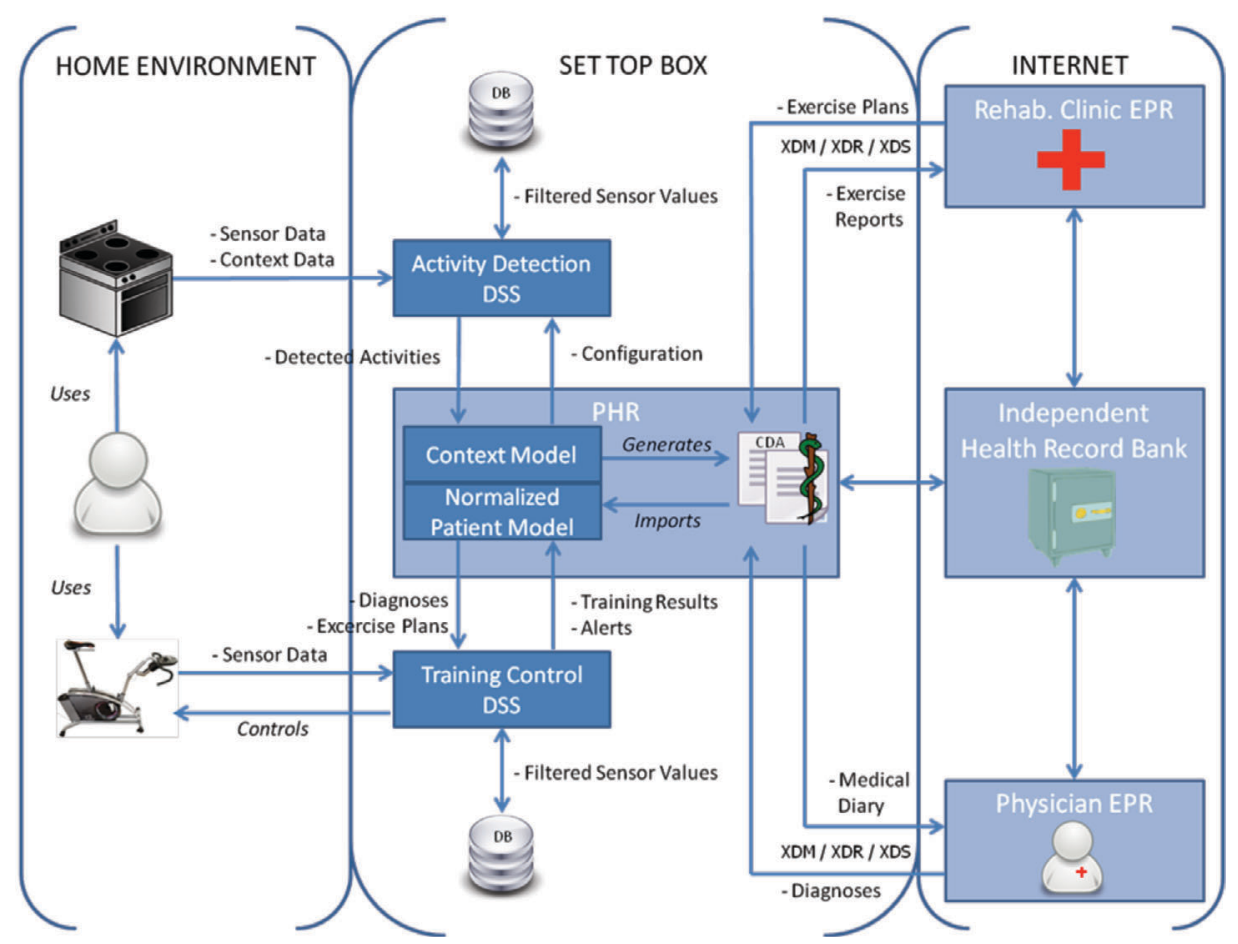

Figure 3. Architectural overview of PHR and related components. Items with leading "-" describe example data; items in italics describe actions.

by decision support systems (DSS). The PHR can be optionally connected with external components such as an independent health record bank, electronic patient record (EPR) or a third party PHR. The interoperability for such data exchange can be facilitated through the use of three Integrating the Healthcare Enterprise (IHE) integration profiles [16], viz. Cross-enterprise Document Media Interchange (XDM), Cross-enterprise Document Reliable Interchange (XDR), and Cross-enterprise Document Sharing (XDS).

\subsection{Implementation of the Prototype}

We have implemented a technical prototype of the PHR system described above, which is localised in a user's home environment. The system delivers and stores data for both medical applications, which have also been implemented as technical prototypes. The PHR and the medical applications are embedded in a middleware platform which contains further AAL related functionality (see Figure 4 and [17]) such as a device abstraction layer or services for the communication of alarms to patient's family members or care personnel.

The components are running inside an "Open Service Gateway initiative" (OSGi) framework [18], a Java based runtime framework for embedded devices. Besides 


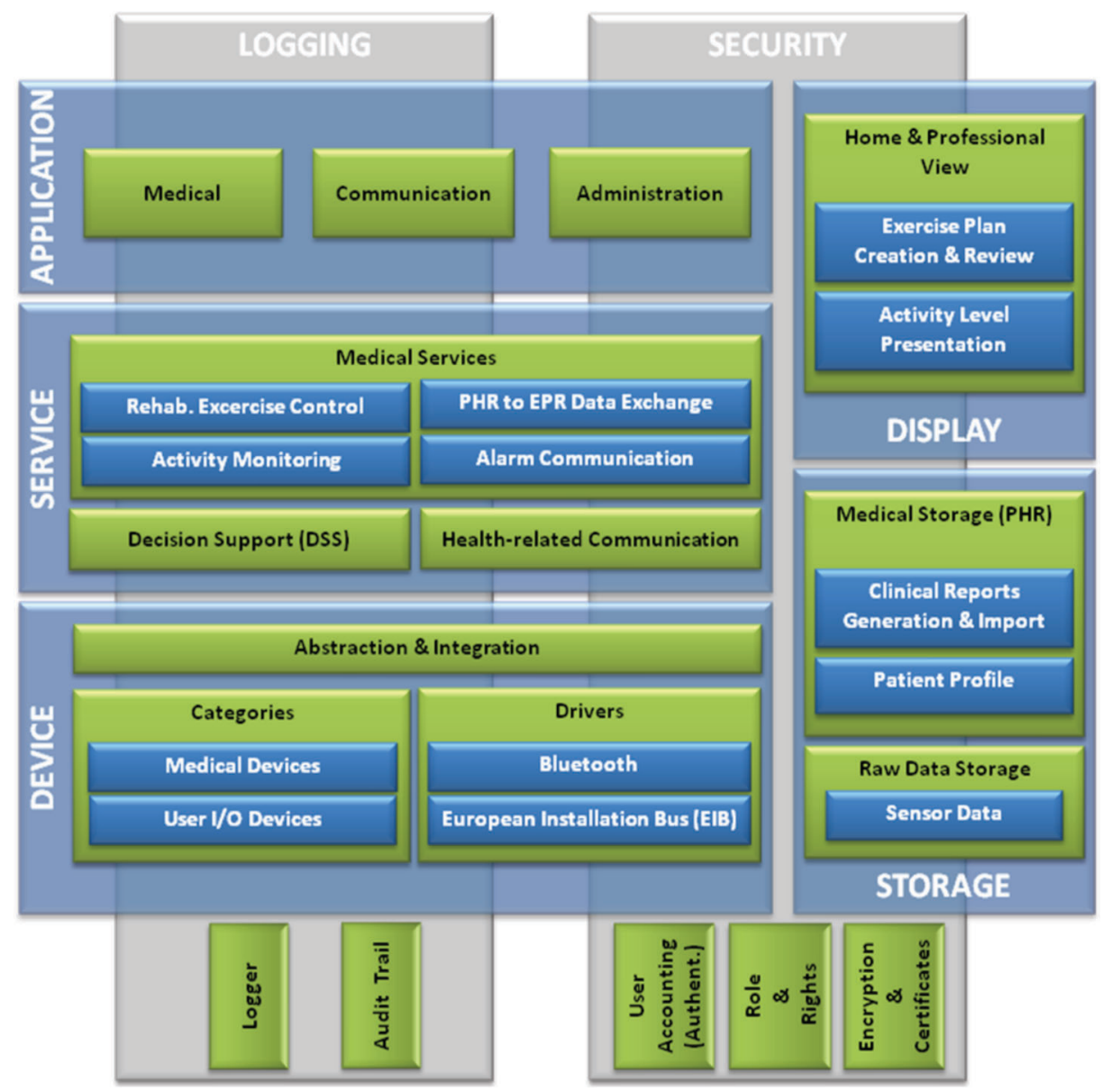

Figure 4. Technical design and components of the AAL platform.

enabling component based development, this framework also allows for standardised provisioning of services including continuous remote updates of services during runtime by means of the Initial Provisioning specification [18, 19]. Additionally, the platform provides essential functionality for configuration (e. g., addresses of communication partners, user variables, etc.) and administration (e.g., user management and component management) of available services.

The PHR uses CDA which has been defined by Health Level Seven (HL7) as a standard document format for the storage and exchange of clinical information. Besides CDA, there are a number of other standards aiming for interoperability between medical applications (e.g., DICOM Structured Reporting [20] and the Continuity of Care Record (CCR) [21]). To date, none of these standards has achieved a significant penetration in the market for health information systems [22]. Both the document- 


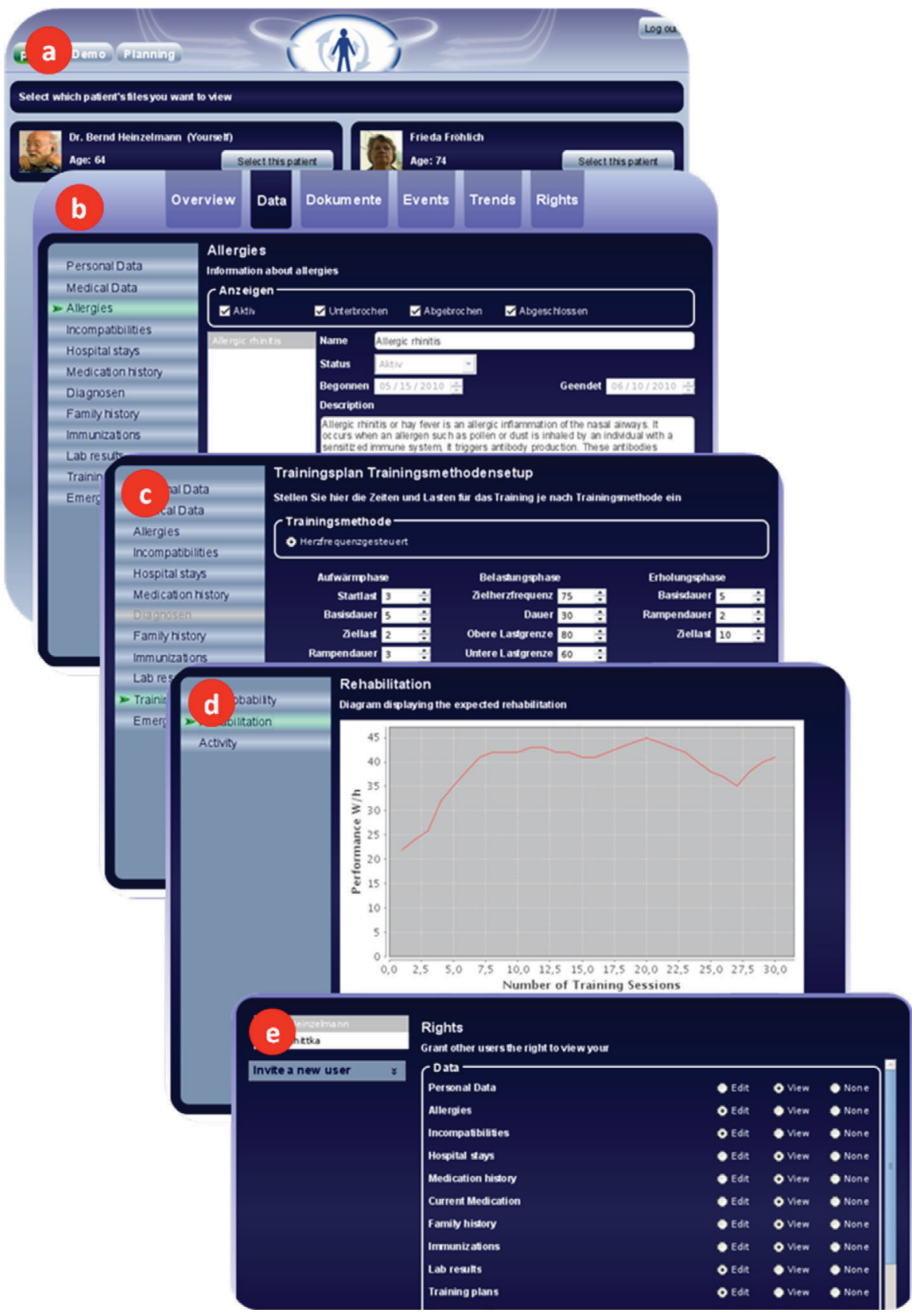

Figure 5. Screenshots of the prototype PHR: (a) Patient selection, (b) Allergies page, (c) Training plan creation, (d) Result trends, (e) Administration of access rights. 
oriented CDA and the message-oriented HL7v3 (the successor of the widely used HL7v2 standard) are derived from the same Reference Information Model (RIM) which makes them highly interoperable [23].

The PHR provides a central storage mechanism for internal components, which assures that a defined set of metadata is included whenever a component stores information in the record. According to the 3DLC model, the medical sensor data should be stored in the PHR. Externally or internally generated clinical documents containing these data are stored as files in the file system. This is useful for larger sensor data collections such as ECG recordings, and enables the PHR to keep incoming original documents, but causes a decrease in performance when components like the DSS perform complex queries such as "select the average heart rate from the last 80 training sessions", where every relevant file has to be opened and parsed. To avoid this, the PHR allows software components to extend the common storage mechanism with data extraction and processing procedures optimised for the specific data items (for example, to load and store the heart rate derived from the ECG in a related database).

\section{DISCUSSION}

The basic functions of the PHR prototype have been implemented and are operational, but the overall assistive system is still under development. The communication standards are not fully implemented yet, but the system is already able to read, write and communicate CDA documents through the usage of an IHE compliant XDM interface.

The present architectural approach has advantages and disadvantages. Applications requiring a high availability of medical and sensor data (such as DSS) are operating locally and do not depend on the availability of an internet connection. Also, it is possible to store data from systems that have no storage capacity (such as sensors for the activity monitoring system). However, if for any reason the internet connection breaks down, the health information is not accessible for external users, and a backup of the data to a spatially separated server cannot be guaranteed. Mobile communication networks can be used as a fallback solution for landline internet connections (or vice versa). Today, many internet routers have this functionality already built in. If there is an external component such as another PHR, or an online backup service, then backup can be delayed until the internet connection is restored.

We currently have no "clinical" counterpart that would be ready to read and process our documents. We are planning a controlled clinical trial with 2000 trainings sessions for patients with COPD. This clinical trial will demonstrate how well the standardised communication of our PHR works. It is anticipated that the standardised integration and exchange of large amounts of sensor data (from ECG, home automation systems, etc.) in clinical documents may need further improvement. Furthermore, we will fine-tune the level of abstraction for the presentation of data to the physicians involved. We are also planning to work on benchmark sensor data quality and on the enhancement of the 3DLC model. To this end, we will integrate more applications, to be developed in other AAL projects, into the PHR prototype through the usage of the 3DLC model. 


\section{CONCLUSION}

The demographic change leads to new types of medical applications which operate in the user's home environment. The wide spectrum of these applications is characterised by a new mix of locations, participants and techniques. Health professionals have to rely on information obtained under new circumstances, which features different quantity and quality of data. A PHR is one way to store, present and communicate such information, so that it becomes available in the professional decision making process. This brings about a number of technical issues with regard to the design of an PHR that can best benefit patient care.

In addressing two different scenarios, a tele-rehabilitation training and an activity detection scheme that is also used to perform new forms of assessments at home, we have created a model for the classification of a wide range of these new medical applications. The 3DLC model presented in this work is one of the first systematic attempts to bring order (in the form of taxonomy) into the somewhat chaotic domain of AAL applications. Furthermore, we have suggested a new architectural approach optimised for the creation of PHRs which can serve as highly available data sources for these applications, and are also compatible with other types of electronic records. Finally, we have outlined further functional and non-functional requirements which should be fulfilled by every PHR system.

The 3DLC Model has enabled us to make some difficult decisions, which will arise in every development process of PHRs intending to support AAL applications. The model has proven to be helpful in drawing a cut-off line in terms of which data should be stored in the PHR and which should not. The model also tackles the problems of the presentation and representation of the sensor data obtained on different abstraction levels. Our PHR prototype has implemented these options and has used appropriate standards wherever possible to ensure maximum interoperability with other healthcare IT systems. We have created a CDA template to fulfil all the needs of the two application scenarios and have implemented an effective retrieval mechanism for documents and sensor data. The service-oriented platform we have chosen allows for the installation and update of further useful components (such as alarm communication) and increases the robustness and flexibility of the system. In summary, the 3DLC model and the PHR prototype presented in this work demonstrate promising solutions to address major challenges in the design of PHRs that are able to support new types of AAL applications.

\section{ACKNOWLEDGEMENTS}

This work is funded in part by the Ministry for Science and Culture of Lower Saxony within the Research Network "Design of Environments for Ageing" (grant VWZN 2420/2524), and in part by the German Ministry of Education and Research within the research projects "PAGE" (grant 01 FC O8044) and "OSAMI-D" (grant 01 IS 08003).

\section{REFERENCES}

[1] Tews H. Von der Pyramide zum Pilz. Demographische Veränderungen in der Gesellschaft. Funkkolleg Altern. 1999, 1:137-48.

[2] Mai R. Die Alten der Zukunft. Eine bevölkerungsstatistische Datenanalyse, $1^{\text {st }}$ ed., Opladen: Leske + Budrich, 2003. 
[3] Ball MJ, Smith C, Bakalar RS. Personal health records: empowering consumers. J Healthc Inf Manag, 2007, 21(1):76-86.

[4] Kaelber DC, Shah S, Vincent A, Pan E, Hook JM, Johnston D, Bates DW, Middleton B. The Value of Personal Health Records. AMIA Annu Symp Proc. 2008, 343-4.

[5] Markle Foundation. Connecting Americans to their Health Care: Final Repo rt-Working Group on Policies for Electronic Information Sharing Between Doctors and Patients. http://www.markle.org/publications/892-connecting-americans-their-health-care-executive-summary. 2004. Last visit 2010.01.27.

[6] Kaelber DC, Jha AK, Johnston D, Middleton B, Bates DW. A research agenda for personal health records (PHRs). J Am Med Inform Assoc, 2008, 15(6): 729-736.

[7] Wood DA. Lifestyle and risk factor management and use of drug therapies in coronary patients from 15 countries. Principal results from EUROASPIRE II Euro Heart Survey Programme. Eur Heart J, 2001, 22(7), 554-572.

[8] Kohlmeyer M, Redaèlli M, Büscher G, Schwitalla B, Lauterbach K, Mayer-Berger W. Nachhaltigkeit in der Sekundärprävention - Sekundärprävention bei Patienten mit Koronarer Herzkrankheit durch Anschlussheilbehandlung und anschließende konzeptintegrierte Nachsorge (SeKoNa). Conf Proc DRV Schriften. 2008, 1:405-5.

[9] Gill TM, Williams CS, Tinetti ME. Assessing risk for the onset of functional dependence among older adults: the role of physical performance. J Am Geriatr Soc, 1995, 43(6):603-609.

[10] Beauchet O, Allali G, Berrut G, Hommet C, Dubost V, Assal F. Gait analysis in demented subjects: Interests and perspectives. Neuropsychiatr Dis Treat, 2008, 4(1):155-160.

[11] Committee On Quality Of Health Care In America and Medicine. Crossing the quality chasm: A new health system for the 21 st century. Institute of Medicine Washington, DC, USA: National Academies Press, 2001.

[12] Deutsches Institut für Medizinische Dokumentation und Information. Internationale Klassifikation der Funktionsfähigkeit, Behinderung und Gesundheit. 2005. http://www.dimdi.de/dynamic/de/klassi/ downloadcenter/icf/endfassung/. Last visit 2011.05.05.

[13] Health Level Seven Internatioinal. HL7 Implementation Guide: CDA Release 2 - Continuity of Care Document (CCD). 2007. http://www.hl7.org/implement/standards/cda.cfm. Last visit 2011.05.05.

[14] Dolin R, Alschuler L, Boyer S, Beebe C, Behlen F, Biron P, Shabo A. Hl7 clinical document architecture, release 2. J Am Med Inform Assoc. 2006, 13(1):30-39.

[15] Frenken T, Gövercin M, Mersmann S, Hein A. Precise Assessment of Self-Selected Gait Velocity in Domestic Environments. Conf. Proc. of the 4th International Conference on Pervasive Computing Technologies for Healthcare 2010. 2010.

[16] IHE International. http://www.ihe.net/. Last visit 2010.01.27.

[17] Hein A, Eichelberg M, Nee O, Schulz A, Helmer A, Lipprandt M. A Service Oriented Platform for Health Services and Ambient Assisted Living. Conf Proc International Conference on Advanced Information Networking and Applications Workshops. 2009, 1:531-6.

[18] The OSGi Alliance. OSGi Service Platform Service Compendium. http://-www.osgi.org/Download/ File?url=/download/r4v42/r4.cmpn.pdf. Last visit 2010.01.27.

[19] Eichelberg M, Hein A, Büsching F, Wolf L. The GAL middleware platform for AAL - a case study. Proc First International Workshop on AAL Service Platforms. 2010, 1:1-6.

[20] Clunie DA. DICOM Structured Reporting, 1st ed., PixelMed Publishing, Bangor PA, 2000.

[21] ASTM. ASTM E2369-05: Standard Specification for Continuity of Care Record (CCR). 2005.

[22] Eichelberg M, Aden T, Riesmeier J, Dogac A, Laleci GB. A survey and analysis of Electronic Healthcare Record standards. ACM Comput Surv. 2005, 37(4):277-315.

[23] Hinchley A. Understanding Version 3: A primer on the HL7 Version 3 Healthcare Interoperability Standard - Normative Edition, 4st ed., Alexander Mönch Publishing, Munich, 2007. 


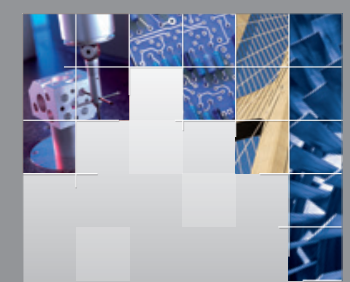

\section{Enfincering}
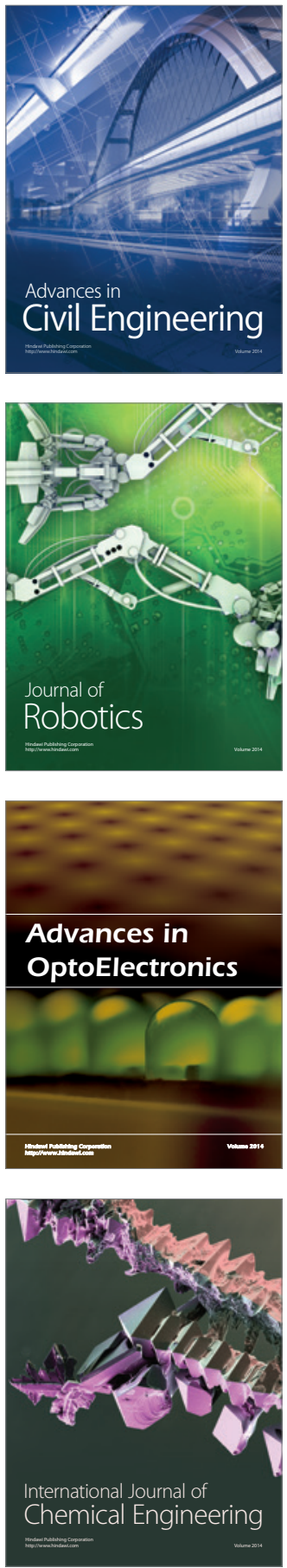

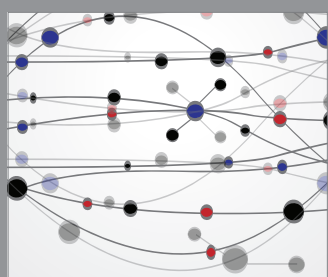

The Scientific World Journal

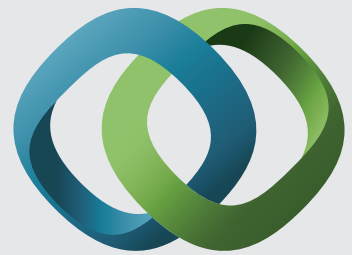

\section{Hindawi}

Submit your manuscripts at

http://www.hindawi.com
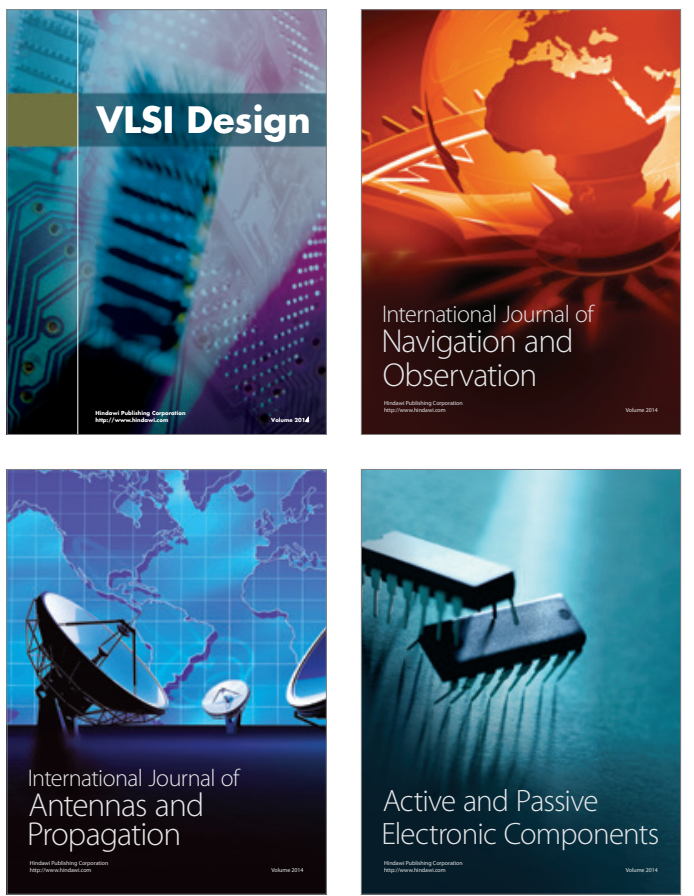
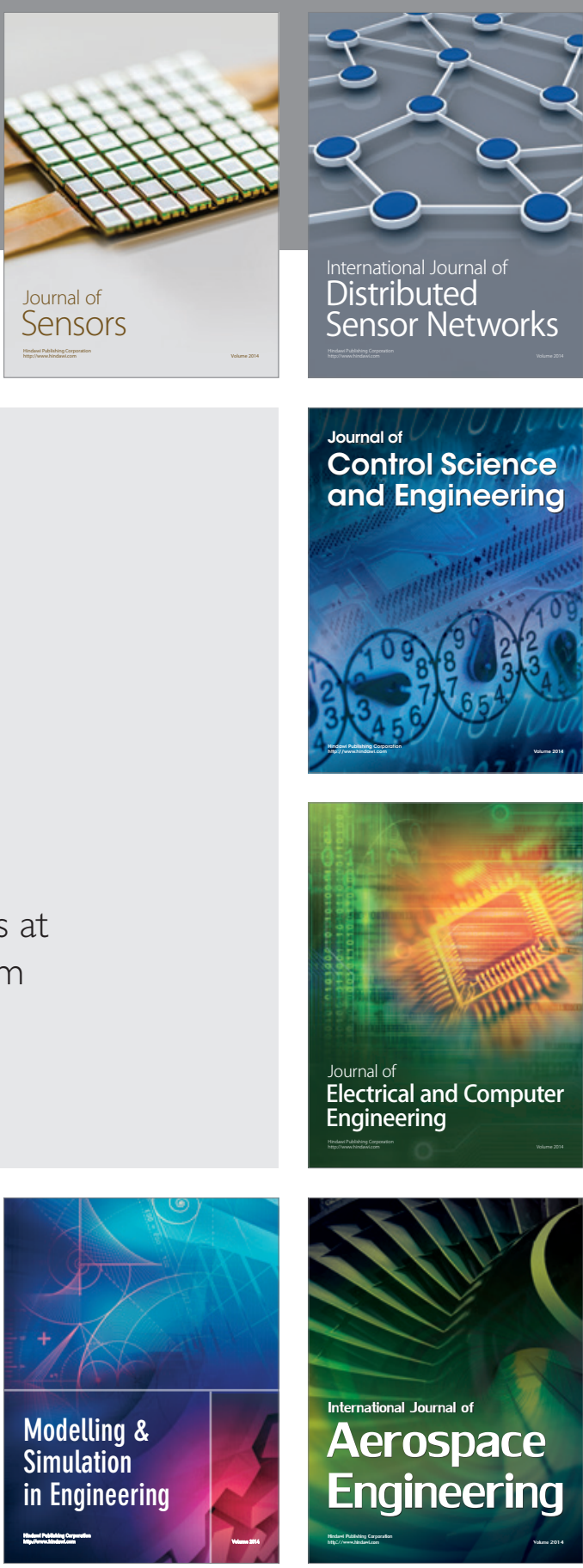

International Journal of

Distributed

Sensor Networks

Journal of

Control Science

and Engineering
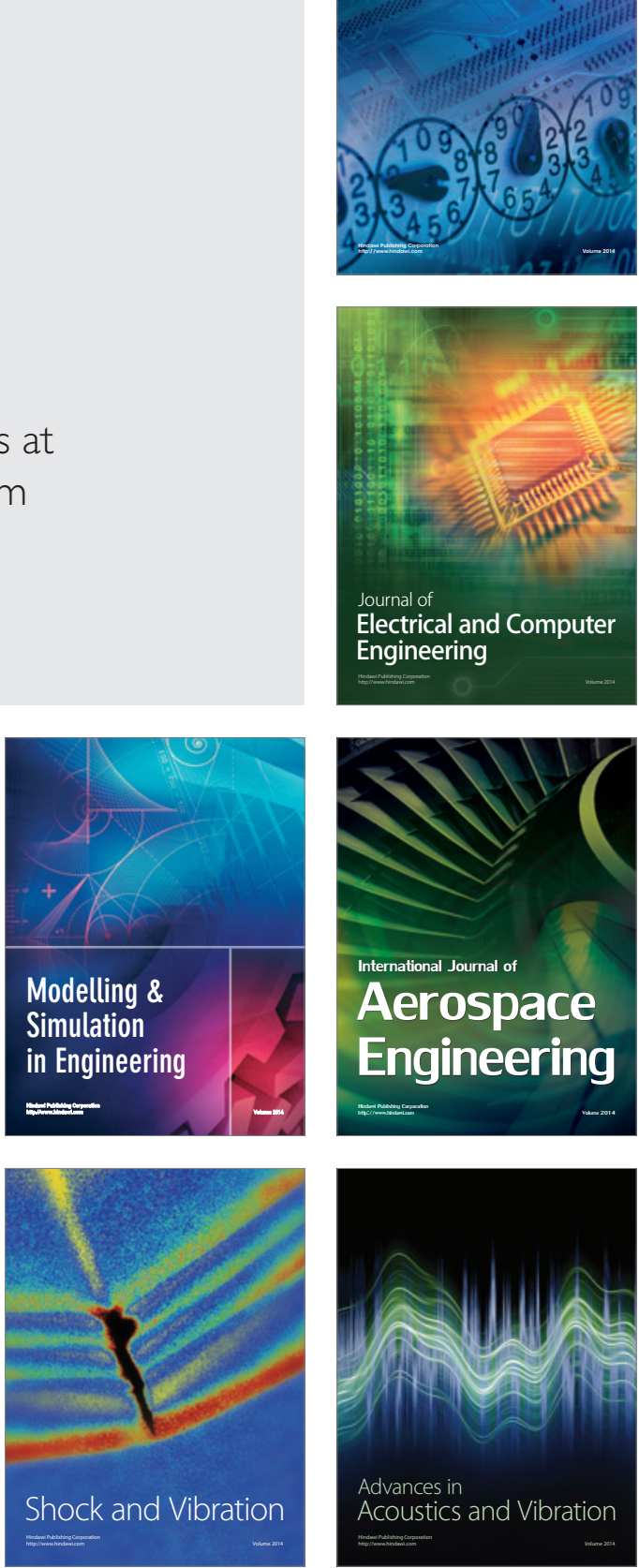